\title{
Smart combinations to prevent HIV rebound
}

\begin{abstract}
Antiretroviral therapy (ART) for HIV prevents the virus replicating, but does not eliminate it. If treatment is stopped, the virus rapidly re-emerges from latent reservoirs in $\mathrm{CD} 4^{+} \mathrm{T}$ cells, an event termed viral rebound, which can progress to AIDS. Three studies have now assessed novel treatment combinations in the absence of ART. In two of these studies, led by Michel Nussenzweig, the combination of two broadly neutralizing antibodies (bNAbs) that target different sites of the viral protein Env maintained long-term suppression. Another study, led by Dan Barouch, showed that the combination of an immune stimulator and a bNAb in monkeys infected with a hybrid of HIV and the simian immunodeficiency virus (SHIV) suppressed viral rebound after ART discontinuation.

In human clinical trials, 3BNC117 (a bNAb that targets the CD4 binding site on the HIV-1 envelope spike)
\end{abstract}

and 10-1074 (a bNAb that targets the base of the V3 loop and surrounding glycans) significantly reduced viraemia. However, this reduction was transient and antibody-resistant viral variants emerged. To determine whether the combination of $3 \mathrm{BNC} 117$ and $10-1074$ is safe and effective in maintaining HIV-1 suppression during interruption of ART, Nussenzweig's group carried out two phase Ib trials.

Mendoza et al. assessed the combination in 11 participants who had complete viral suppression after ART; 9 maintained viral suppression for a median of 21 weeks compared with 6-10 weeks of viral suppression with monotherapy in historical controls. In the two participants that rebounded early, their pretreatment latent reservoir harboured 3BNC117resistant or 10-1074-resistant viruses.

In turn, Bar-On et al. analysed the antiviral activity of the combination in viraemic individuals. The combination of 3BNC117 and 10-1074 was more effective in suppressing viraemia than either antibody alone. Of note, antibody half-lives were lower in viraemic individuals than in individuals on ART with suppressed viral loads. The authors concluded that whereas two antibodies may be sufficient in individuals with very low levels of viraemia, additional therapies would be required for viraemic individuals. To this end, the authors are now developing longer half-life versions of their antibodies.

Borducchi et al. assessed the combination of another bNab, PGT121, and GS-9620 (vesatolimod), an agonist of Toll-like receptor 7 (TLR7), which activates immune cells to destroy virus-infected cells. The authors infected 44 monkeys with SHIV. A week later, the animals began 130 weeks of ART. HIV rapidly disappeared from the blood of all monkeys and after 96 weeks, the monkeys were randomly assigned to receive one of four additional treatments for 18 weeks: no intervention, GS-9620, PGT121 or both compounds. After stopping ART, viral rebound was identified in all 11 animals that received no intervention and in 10 and 9 of the animals that received GS-9620 or 3BNC117, respectively. By contrast, only 6 of the 11 monkeys treated with both GS-9620 and PGT121 showed viral rebound by week 28 after ceasing ART. The other 5 monkeys in this group remained clear of any detectable virus.

Mechanistically, the authors found that $\mathrm{CD} 4^{+} \mathrm{T}$ cells and natural killer cells were activated in all monkeys that received GS-9620 and propose that GS-9620 treatment activates latently infected $\mathrm{CD} 4^{+} \mathrm{T}$ cells, rendering them more susceptible to PGT121 binding, thus facilitating antibody-mediated elimination of the infected $\mathrm{CD} 4^{+} \mathrm{T}$ cells.

These papers present interesting strategies to prevent viral rebound and, in the case of Borducchi et al., to target the latent reservoir. The biggest test will now be whether GS 9620 and PGT121 can produce similar results in people.

M. Teresa Villanueva

ORIGINAL ARTICLES Mendoza, P. et al. Combination therapy with anti-HIV-1 antibodies maintains viral suppression. Nature 561, 479-484 (2018) | Bar-On, Y. et al. Safety and antiviral activity of combination HIV-1 broadly neutralizing antibodies in viremic individuals. Nat. Med. https:// doi.org/10.1038/s41591-018-0186-4 (2018) | Borducchi, E. N. et al. Antibody and TLR7 agonist delay viral rebound in SHIV-infected monkeys.

Nature https://doi.org/10.1038/

s41586-018-0600-6 (2018)

FURTHER READING Ferrari, G. et al. Envelopespecific antibodies and antibody-derived molecules for treating and curing HIV infection. Nat. Rev. Drug Discov. 15, 823-834 (2016) 